\title{
Gestão do cuidado a usuários com feridas crônicas na Atenção Básica
}

\section{Care management to chronic wound carriers in Primary Health Care}

\author{
Denis Fernandes da Silva Ribeiro'
}

\begin{abstract}
RESUMO
Objetivo: Caracterizar o perfil dos usuários portadores de lesões crônicas e o perfil de cuidados implementados a estes sujeitos por uma Unidade Básica de Saúde com Estratégia Saúde da Família. Metodologia: Pesquisa documental, descritiva, exploratória e de abordagem quantitativa em que se estudou dados referentes ao processo de cuidado a portadores de feridas crônicas em uma Unidade Básica de Saúde. Foram analisados, retrospectivamente, dados de agosto de 2017 a julho de 2018 . Resultados: O público era predominantemente idoso e discretamente feminino, no qual a hipertensão arterial sistêmica, o diabetes mellitus e as doenças vasculares periféricas, foram doenças crônicas prevalentes como diagnóstico principal. Do total, 56,7\% apresentavam lesões vasculares, que em 46,7\% exibiam tecido de granulação e $63,3 \%$ manifestava infecção ou colonização. O cuidado foi realizado em $96,7 \%$ dos casos nos consultórios e na sala de curativos da unidade de saúde. A maior parte das coberturas especiais $(62,5 \%)$ esteve ausente em algum intervalo de tempo no período estudado. Conclusões: Evidenciou-se a necessidade de fortalecimento do papel da atenção básica no manejo das lesões crônicas, bem como a relevância do abastecimento efetivo de insumos para redução da fragmentação do cuidado.
\end{abstract}

Palavras chave: Ferimentos e Lesões. Casos Crônicos. Atenção Primária à Saúde.

\begin{abstract}
Objective:To characterize the profile of users with chronic injuries and the care profile implemented for these users by a Basic Health Unit with Family Health Strategy. Methodology: This is a documentary, descriptive, exploratory research and quantitative approach in which data related to the care process to chronic wounds carriers in a Basic Health Unit were studied. Data from August 2017 to July 2018 were retrospectively analyzed. Results:The public was predominantly elderly and discreetly female, in which systemic arterial hypertension, diabetes mellitus and peripheral vascular diseases were prevalent chronic diseases as the main diagnosis. Of total, $56.7 \%$ had vascular lesions, which in $46.7 \%$ had granulation tissue and $63.3 \%$ showed infection or colonization. The care was performed in $96.7 \%$ of cases in the medical rooms and rooms of bandages of the health unit. Most of the special healings $(62.5 \%)$ was absent at some time during the study period. Conclusions: The need to strengthen the role of primary care in the management of chronic injuries was evidenced, as well as the relevance of the effective supply of inputs to reduce care fragmentation. Keywords: Wounds and Injuries. Chronic Disease. Primary Health Care.
\end{abstract}


INTRODUÇÃO

AAtenção Básica $(A B)$ é, hoje, a porta de entrada prioritária e um dos pilares de sustentação do Sistema Único de Saúde (SUS), considerando o seu potencial para reorientação do modelo de atenção à saúde e para o fortalecimento dos princípios do SUS da universalidade, equidade e integralidade. É um nível de atenção que se operacionaliza através de ações territorializadas, centradas no sujeito e que almejam a resolutividade através da longitudinalidade e da coordenação do cuidado(1-3).

$\mathrm{Na}$ última década a $A B$ tem proporcionado melhorias à condição de saúde da população, com o aumento da oferta/ cobertura de saúde, diminuição das desigualdades sócio sanitárias e diminuição da morbimortalidade (sobretudo infantil) ${ }^{(2-4)}$. Apesar disso, ainda enfrenta a interposição de inúmeros desafios ao seu funcionamento, que distanciam o alcance da resolutividade proposta ${ }^{(5)}$.

$A$ potencialidade de resolução da $A B$ não tem sido efetivada, pois além de necessitar de recursos humanos, materiais e estruturais plenos e articulados em redes intra e intersetoriais, a $A B$ tem o desafio de atuar frente aos riscos e vulnerabilidades sociais ${ }^{(1,4)} \mathrm{em}$ um cenário de caos em que nem sempre os serviços dispõem de ferramentas e estruturação para tal( ${ }^{(4,5)}$.

As feridas crônicas são situações (casos) que podem ser utilizadas como narrativa de análise das ferramentas, da estruturação e do funcionamento da $A B$, tendo em vista a sua alta prevalência nestes serviços ${ }^{(6-8)}$. Isto decorre, pois a $A B$ é o nível de assistência preferencial para manejo das Doenças Crônicas Não Transmissíveis (DCNT) ${ }^{(5,7,9)}$, principal etiologia das feridas e lesões crônicas ${ }^{(10-12)}$, dois termos que serão utilizados neste trabalho como sinônimos.

Dada a fase de transição epidemiológica que se vive nos dias atuais, com a maior prevalência de DCNT como causa de morbimortalidade da população( ${ }^{(5)}$, a $A B$ tem a responsabilidade de acompanhar longitudinalmente tal grupo de usuários, atuando especialmente na prevenção do adoecimento, no diagnóstico precoce, na prevenção de complicações e iatrogenias ${ }^{(1,5,7,8)}$.

Entretanto, por variados motivos, é frequente a ocorrência de feridas como complicações dos agravos crônicos, fazendo com que estas se configurem como relevante problema de saúde pública ${ }^{(13-15)} \mathrm{em}$ especial, quando são analisados os graus de incapacidades aos sujeitos, além dos impactos psicossociais e econômicos ${ }^{(8,16,17)}$.

Embora inexistam bases de dados com estatísticas sobre a prevalência de lesões de pele na população ${ }^{(12,14,15)}$, posto que o subregistro é uma descontente realidade no Brasil, estima-se que as lesões de pele ocorram em $3 \%$ da população(6) sendo que a incidência e a prevalência são maiores na população que vive com diabetes mellitus, vasculopatias periféricas e outras DCNT(10-12,16,17).

Nos usuários portadores de DCNT, as feridas tem maior potencial de cronificação, tornando-se feridas crônicas, que atualmente recebem a denominação de feridas complexas ${ }^{(18)}$. Por definição, as feridas crônicas ou complexas são aquelas refratárias ao tratamento e que têm longa duração, com curso superior a 30 dias, assim como aquelas com recorrência

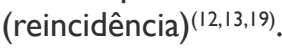

As incapacidades geradas pelas lesões crônicas aos sujeitos vão além de questões laborais, incluem-se limitações físicas que são dolorosas e dificultam a deambulação, impactam negativamente à qualidade de vida(9,10). Há que se mencionar, também, ○ grau de sofrimento psíquico dos portadores destas lesões, sendo comuns alterações na autoimagem e no autoconceito, propiciando processos crônicos de baixa autoestima, além do desenvolvimento de transtornos de humor e isolamento social ${ }^{(6,10,16)}$.

Sobre os impactos econômicos, é necessário pontuar que os cuidados às feridas crônicas geram destacável ônus financeiro tanto ao usuário, quanto ao sistema de saúde ${ }^{(19)}$. Os insumos materiais necessários para este fim têm alto custo e, por serem onerosos, têm quantidade finita e por vezes escassa nos serviços ${ }^{(6,15)}$.A assistência a este público demanda, ainda, a mobilização de recursos humanos especializados para a utilização das tecnologias de cuidado com efetividade ${ }^{(6)}$, contudo, no cotidiano dos serviços, é comum a presença de alta carga de trabalho, geralmente associada a desorganização dos fluxos e processos de trabalho, que impactam diretamente no resultado da assistência ${ }^{(9)}$.

Tendo como pressuposto que os serviços de $A B$ despontam como um novo dispositivo para o manejo das feridas crônicas, em substituição ao hegemônico modelo hospitalar e que a organização e a estruturação destes serviços de cuidados primários são essenciais para a otimização do tempo de cicatrização, promoção de maior qualidade de vida e redução do ônus ao sistema de saúde, este estudo se faz relevante pois desvela variáveis associadas a assistência aos sujeitos com feridas crônicas em um cenário não muito explorado na literatura da temática, as Unidades Básicas de Saúde (UBS).

O conhecimento sobre o perfil de pacientes que utilizam uma UBS como fonte de cuidado para tratamento de suas lesões crônicas, bem como das ofertas de cuidado desse serviço podem gerar dados essenciais para a elaboração de diretrizes que fortaleçam $\circ$ papel da $A B$ no manejo destas condições.

Deste modo, são objetivos: caracterizar o perfil dos usuários portadores de lesões crônicas e o perfil de cuidados implementados a estes sujeitos por uma Unidade Básica de Saúde; e analisar a oferta e a demanda de recursos materiais para manejo das lesões na Unidade Básica de Saúde estudada.

\section{METODOLOGIA}

Trata-se de uma pesquisa documental, descritiva, exploratória e de abordagem quantitativa em que se estudou dados referentes ao processo de cuidado a portadores de feridas crônicas em uma Unidade Básica de Saúde (UBS) com Estratégia Saúde da Família (ESF). Esta UBS é localizada em um bairro periférico do município do Rio de Janeiro/RJ e contava com cinco Equipes de Saúde da Família (EqESF) completas, que eram responsáveis pelo acompanhamento de uma população de 16.500 usuários.

Para a seleção da amostra de usuários com feridas crônicas foram adotados os seguintes critérios de inclusão:ser portador de lesão de pele, acompanhar uma mesma lesão no serviço estudado por tempo igual ou superior a trinta dias; e o critério de exclusão: casos em que o acompanhamento era irregular, isto é, aqueles casos que havia intervalo superior a trinta dias entre os registros. A amostra final consistiu em trinta usuários.

Como fonte de dados, foram utilizados relatórios de gestão fornecidos pelo prontuário eletrônico (PEP) implementado na unidade, e, em especial, as planilhas em Microsoft Excel [ de acompanhamento dos usuários portadores de feridas. As variáveis levantadas dos relatórios de gestão do PEP foram 
idade, sexo, diagnóstico principal, morbidades associadas, número de curativos registrados, número de procedimentos registrados para cada usuário, profissional que originou cada registro, além da categoria profissional deste. Das planilhas de acompanhamento foram coletados os dados sobre as variáveis: tempo de duração, localização, etiologia, microbiologia, tipo de tecido predominante nas lesões, assim como as informações sobre utilização e gerenciamento de insumos materiais. Os dados foram analisados retrospectivamente a partir do recorte temporal de agosto de 2017 a julho de 2018 .

Os dados levantados foram inseridos em um banco de dados em Microsoft Excel $\circledast$, que foi trabalhado através do software Epi InfoTM 7.2.3 do Centers for Disease Control and Prevention (CDC), por onde se realizou análise estatística descritiva com o levantamento de frequências simples e combinadas e tabulações cruzadas.

O estudo fez parte da pesquisa "A gestão do cuidado na abordagem de casos complexos no âmbito da Estratégia Saúde da Família" realizada no curso de Residência Multiprofissional em Saúde da Família da Escola Nacional de Saúde Pública Sergio Arouca (ENSP/FIOCRUZ). As recomendações das Resoluções $n^{\circ} 466 / 12$ e $n^{\circ}$ 510/16 do Conselho Nacional de Saúde foram cumpridas, onde a pesquisa foi apreciada e aprovada pelos Comitês de Ética em Pesquisa da ENSP/FIOCRUZ, sob o parecer 2.778.139/2018 e da Secretaria Municipal de Saúde do Rio de Janeiro, sob o parecer 2.856.7I7/20I8.

\section{RESULTADOS E DISCUSSÃO}

O público de usuários crônicos do setor de curativos era discretamente feminino, com representação em 53,3\% dos casos por mulheres, e formado, majoritariamente, por idosos $(66,7 \%)$, onde a idade média foi 65,3 anos e a mediana foi 65,5 anos. Quando se compara a outra parcela dos usuários, que é composta por adultos com idade entre 40 e 59 anos, notase que parte considerável da população afetada pelas feridas crônicas $(33,3 \%)$ encontrava-se em idade economicamente produtiva.

Tabela I - Caracterização dos usuários portadores de feridas crônicas em uma UBS da cidade do Rio de Janeiro/RJ, entre agosto de 2017 e julho de 2018 .

\begin{tabular}{|l|c|c|}
\hline Características & $\mathbf{n}$ & $\%$ \\
\hline Sexo & 16 & 53,3 \\
\hline Feminino & 14 & 46,7 \\
\hline Masculino & 4 & 13,3 \\
\hline Faixa etária & 6 & 20,0 \\
\hline 40 a 49 anos & 9 & 30,0 \\
\hline 50 a 59 anos & 5 & 16,7 \\
\hline 60 a 69 anos & 5 & 16,7 \\
\hline 70 a 79 anos & 1 & 3,3 \\
\hline 80 a 89 anos & 10 & 33,3 \\
\hline$\geq$ a 90 anos & 10 & 33,3 \\
\hline Diagnóstico principal & 5 & 16,7 \\
\hline Diabetes mellitus tipo 2 & 2 & 6,7 \\
\hline Hipertensão arterial & \\
\hline Doenças vasculares periféricas &
\end{tabular}

\begin{tabular}{|c|c|c|}
\hline Desnutrição & 1 & 3,3 \\
\hline Etilismo & 1 & 3,3 \\
\hline Obesidade & $\mathrm{I}$ & 3,3 \\
\hline \multicolumn{3}{|c|}{ Tempo duração da lesão } \\
\hline$\geq 01$ a 06 meses & 10 & 33,3 \\
\hline 07 a 12 meses & 1 & 3,3 \\
\hline 02 a 04 anos & 4 & 13,3 \\
\hline 05 a 07 anos & 7 & 23,3 \\
\hline 08 a 10 anos & 3 & 10,0 \\
\hline$\leq \mathrm{a} \| \mathrm{I}$ anos & 1 & 3,3 \\
\hline Sem informação & 4 & 13,3 \\
\hline
\end{tabular}

Fonte: Dados da pesquisa, com elaboração própria pelo autor.

Quanto a situação de saúde dos sujeitos, por meio da tabela I, verifica-se que $83 \%$ tinha o diagnóstico principal de hipertensão arterial sistêmica (HAS), diabetes mellitus tipo 2 (DM) e/ou de doenças vasculares periféricas (DVP). Na literatura existem resultados semelhantes, com maior frequência de feridas em mulheres ${ }^{(8,10,13)}$, com pico de ocorrência a partir dos 60 anos $^{(8,14,15)}$, com média de idade \pm 64 anos $^{(12)}$.

Assim como encontrado neste estudo, na literatura houve associação direta entre a ocorrência de DCNT e feridas crônicas ${ }^{(8,10,13)}$, em que a HAS, o DM e as DVP foram prevalentes. Interessante ressaltar que a relação entre DCNT e as feridas crônicas a partir dos 60 anos decorre do processo de redução das reservas fisiológicas e alterações no processo de reparação tecidual associadas ao envelhecimento $(8,14)$. Tornando, portanto, primordial o monitoramento prévio e pregresso ao desenvolvimento de feridas nos usuários portadores de DCNT, principalmente idosos, considerando o alto risco de cronificação destas.

O tempo de duração das feridas era prolongado, posto que em $49,9 \%$ dos casos o tempo de duração superava 02 anos, sendo que existiam usuários que portavam uma mesma ferida por mais de II anos. A cronificação das lesões é produto da interação de vários fatores intrínsecos e extrínsecos, tais como idade, nutrição, carga de doenças, etiologia da lesão, adesão ao tratamento, nível educacional, renda, trabalho, moradia, além do acesso aos recursos e serviços de saúde $(6,8,11,12,15)$.

Do total de usuários $76,6 \%(n=23)$ apresentava associações de duas morbidades e $63,3 \%(n=19)$ tinha associação de três morbidades. O risco social ocorreu em $23,3 \%$ dos casos, revelando-se como um fator determinante na prevalência de lesões e cronificações destas.As morbidades que acometem os portadores de feridas crônicas, isto é, os agravos que não eram o diagnóstico principal, são especificadas na figura I. Nota-se que a carga de comorbidades era alta e que estas conseguiam influenciar negativamente no processo cicatricial das feridas. 
Figura I - Comorbidades em portadores de feridas crônicas usuários numa UBS do município do Rio de Janeiro, entre agosto de 2017 e julho de 2018 .

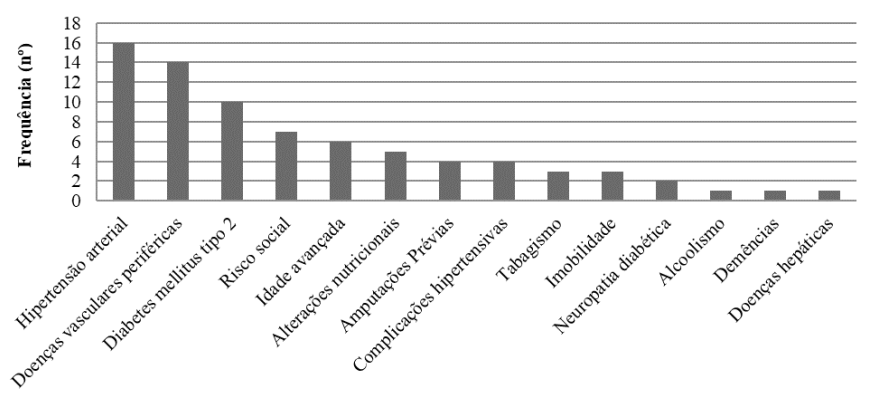

Fonte: Dados da pesquisa, com elaboração própria pelo autor.

Cabe mencionar que além das conhecidas DCNT e suas complicações, um resultado interessante foi a consideração do "risco social" como um fator que exercia influência nas lesões crônicas. O contato capilarizado com o espaço onde acontece a vida das pessoas - o território - possibilita uma visão ampliada sobre os determinantes e condicionantes sociais que afetam a população(1,4,5) e, portanto, essa territorialização, possível na $A B$, permitiu considerar o risco social como um fator interveniente da cronificação das lesões, o que nem sempre seria identificável em outro nível de atenção à saúde.

No intervalo de tempo estudado, foram registrados 279 curativos, evidenciando uma média de 9,3 curativos/ usuário ao ano, ou seja, ao se considerar o período de OI ano, houve menos de um procedimento por usuário ao mês. Entretanto, os relatórios analisados permitem levantar duas hipóteses: a ocorrência de subregistro e a ocorrência de hiperacompanhamento de determinados usuários, em detrimento de outros.

Dos procedimentos registrados, 61,6\% $(n=172)$ foram realizados por profissional técnico de enfermagem e os demais por enfermeiro. Não houve qualquer registro sobre a realização de curativo ou avaliação de ferida por outro profissional da saúde. Interessante pontuar que de todos os registros realizados pelo técnico de enfermagem, aproximadamente $54 \%$ $(n=I 50)$ tiveram origem de um único profissional, sendo que a unidade dispunha de cinco profissionais técnicos que não eram fixos aos setores e trabalhavam em regime de escala, indicando, desta maneira, que o registro dos procedimentos não era rotina dos profissionais de nível técnico, corroborando, assim, a hipótese de subregistro.

Embora os registros associados as lesões crônicas sejam uma ferramenta substancial para avaliar e monitorar o tratamento, além de mensurar a custo efetividade e accountability das ações e dos insumos, a ausência e inadequação dos registros do cuidado têm sido reportadas como um problema comum no cotidiano dos serviços de saúde ${ }^{(9,12,15,19)}$. Na perspectiva de alguns autores ${ }^{(9,12,18)}$, essa falta de apropriação da prática do registro ocorre devido a extenuante carga de trabalho, pela indisponibilidade de instrumentos e condições para o registro dos atendimentos, além da desorganização dos fluxos e rotinas de trabalho.

O baixo número de curativos registrados pelos enfermeiros pode indicar não só subregistro dos procedimentos realizados, mas que estes profissionais estivessem priorizando as reavaliações ao invés da realização da técnica dos curativos. Por se tratarem de feridas com evoluções lentas, o número de reavaliações será sempre inferior ao número de curativos realizados.

Ressalta-se que não existia rotina estabelecida no serviço quanto à frequência de realizações dos curativos e das reavaliações, ficando a cargo do profissional de referência enfermeiro da EqESF a que o usuário é vinculado - a definição. Os usuários também exerciam controle na frequência de realizações de curativos, posto que se utilizavam do mecanismo da demanda espontânea como ferramenta de acesso ao atendimento na sala de curativos, isto colaborou com o surgimento de usuários hiperutilizadores.

Foi verificado que cinco usuários $(16,6 \%)$ concentraram $52,3 \%$ do total de procedimentos registrados. Este fenômeno tinha relação com o grau de complexidade da lesão, mas também foi influenciado pela facilidade de acesso a determinados usuários em detrimento de outros, tendo em vista que estes usuários foram os que mais demandaram a realização de curativos e consultas de avaliação à unidade sem agendamento prévio (demanda espontânea). As avaliações e os curativos aconteceram em $96,7 \%$ dos casos nos consultórios e na sala de curativos da unidade, somente em um caso $(3,3 \%)$, os curativos eram realizados através de visitas domiciliares. Não havia rotina e tampouco registros de busca ativa para a ocorrência de faltas ou de abandono de tratamento.

O perfil das lesões foi outra dimensão levantada, em que se identificou a maior parte das feridas acometendo os membros inferiores, com etiologia variada, embora, frequentemente, de origem venosa, com predomínio de tecidos de granulação e de tecidos desvitalizados em leito da lesão e que em $63,3 \%$ dos casos havia evidência clínica ou laboratorial de colonização ou infecção.

Tabela 2 - Identificação das características de feridas crônicas acompanhadas por uma UBS da cidade do Rio de Janeiro/RJ, entre agosto de 2017 e julho de 2018 .

\begin{tabular}{|c|c|c|}
\hline Características & $\mathbf{n}$ & $\%$ \\
\hline \multicolumn{3}{|l|}{ Localização } \\
\hline Membros inferiores & 28 & 93,3 \\
\hline Mama & I & 3,3 \\
\hline Região sacral & 1 & 3,3 \\
\hline \multicolumn{3}{|l|}{ Etiologia } \\
\hline Venosa & 12 & 40,0 \\
\hline Diabética (pé diabético) & 5 & 16,7 \\
\hline Mista (arterial e venosa) & 5 & 16,7 \\
\hline Traumática & 4 & 13,3 \\
\hline Infecciosa & 3 & 10,0 \\
\hline Lesão por pressão & 1 & 3,3 \\
\hline \multicolumn{3}{|l|}{ Tecido predominante } \\
\hline Granulação & 14 & 46,7 \\
\hline Tecido desvitalizados & 12 & 40,0 \\
\hline Epitelização & 4 & 13,3 \\
\hline \multicolumn{3}{|l|}{ Microbiologia } \\
\hline Colonizada & 12 & 40,0 \\
\hline Limpa & 11 & 36,7 \\
\hline Infectada & 7 & 23,3 \\
\hline \multicolumn{3}{|l|}{ Antissepsia da lesão } \\
\hline Solução aquosa de PHMB + SF a 0,9\% & 22 & 73,3 \\
\hline SF a $0,9 \%$, somente & 8 & 26,7 \\
\hline
\end{tabular}

Fonte: Dados da pesquisa, com elaboração própria pelo autor 
A alta frequência de lesões vasculares $(56,7 \%)$ em membros inferiores $(93,3 \%)$ é encontrada em outras investigações realizadas em serviços de $A B$, indicando que estas são características prevalentes das lesões crônicas neste nível de atenção à saúde ${ }^{(8,12)}$. As lesões vasculares decorrem do comprometimento dos vasos sanguíneos e linfáticos que afetam a perfusão e as trocas orgânicas e geram morte celular ${ }^{(6,11)}$. São lesões com potencial cronificante, pois a manutenção da vida celular e a migração das células de defesa, fundamentais para o reparo tecidual, são continuamente dificultadas ${ }^{(13,16)}$.

Dentre as lesões vasculares (venosas, arteriais e mistas), as venosas são as mais prevalentes na população de um modo geral $^{(6,8,11-13,18)}$, o que foi confirmado no cenário aqui estudado, onde estas responderam por $40 \%$ do total. Outro aspecto corroborado pela literatura foi que, como os membros inferiores são as regiões que mais sofrem incompetências vasculares, estes têm a maior propensão ao desenvolvimento de lesões ${ }^{(10,11,13)}$.

A maior parte das feridas (60\%) apresentavam tecidos propícios a cicatrização, com predominância do tecido de granulação, e, em seguida, o tecido de epitelização. Seria algo positivo, entretanto, o tecido de granulação - presente em $46,7 \%$ das lesões - é uma das características patognomônicas das úlceras venosas ${ }^{(13)}$, revelando que provavelmente estas lesões estão estacionadas na fase proliferativa, ao invés de estarem se encaminhando para a fase de maturação, com predomínio de tecido de epitelização. Como é mostrado na tabela 2 , apenas $13,3 \%(n=4)$ das lesões chegaram a apresentar tecido de epitelização no leito da lesão.

No estudo realizado por Vieira e colaboradores $^{(8)}$, o tecido de granulação foi o mais frequente $(48,2 \%)$ no leito de 339 feridas de etiologia variada. Barros e colaboradores ${ }^{(15)}$ encontraram resultado semelhante, com grande prevalência de tecido de granulação $(42,8 \%)$, assim como no estudo de Vieira e Araujo ${ }^{(16)}$, onde foi encontrado em uma proporção de $29,6 \%$. Indicando ser o tecido mais frequente encontrado em lesões crônicas.

Uma ferida, em seu percurso natural, atravessa três fases, que compõem o que se conhece por processo cicatricial. Esse processo é composto por fases sequenciais e interdependentes que são as fases inflamatória, proliferativa e maturação $(6,20)$. Por variados fatores, já anteriormente citados, uma ferida pode estacionar em uma destas fases e não progredir para a cicatrização ou regeneração. Baseando-se nas fases de reparação é possível afirmar que $46,7 \%$ das lesões aqui estudadas encontram-se estacionadas na fase proliferativa, $40 \%$ na fase inflamatória e apenas $13,3 \%$ na fase de maturação.

As lesões aqui estudadas tinham, em $63,3 \%$ dos casos, evidência clínica ou laboratorial de infecção ou colonização. $\mathrm{Na}$ literatura da temática, embora não fossem mencionados dados quantitativos sobre a frequência de infecção e colonização em feridas crônicas, a infecção e a colonização são mencionadas como um importante fator que colabora com a estagnação do processo cicatricial|(6-8,11,13,15,17,18,20).

O número de feridas em que os tecidos desvitalizados foram predominantes correspondeu a $40 \%$ do total. Entende-se por tecidos desvitalizados àqueles que impedem a cicatrização (esfacelos e necroses) e que devem sofrer remoção por meios autolíticos, químicos e instrumentais ${ }^{(6,18,20)}$, visto que a permanência destes tecidos proporciona um processo de inflamação crônica progressiva ${ }^{(6,20)}$. As substâncias e coberturas com ação farmacológica são indispensáveis para intervenção nestas situações em que há estagnação do processo de reparo tecidual.

Sobre as substâncias utilizadas para tratamento da ferida, neste estudo elas são compreendidas e trabalhadas como dois grupos distintos: substâncias para antissepsia e coberturas especiais.Ambos grupos exercem ação terapêutica nas feridas, entretanto o primeiro é utilizado para remover sujidades, restos celulares, exsudatos aderidos na lesão além do controle microbiológico químico ou mecânico ${ }^{(6,18,19)}$; e o último é empregado para manejar a lesão de diferentes maneiras com o objetivo final de propiciar um ambiente propicio a cicatrização(6,18,19).

A antissepsia da lesão foi realizada em $73,3 \%$ das vezes através da combinação do uso de cloreto de sódio (soro fisiológico) a 0,9\% e solução aquosa de polihexanida (PHMB), como exposto na tabela 2. Como $63,3 \%$ das lesões eram colonizadas ou infectadas, entende-se que o uso de PHMB foi efetivo na maior parte das vezes, tendo em vista seu potencial antimicrobiano ao biofilme da lesão( ${ }^{(20)}$. A PHMB foi o insumo terapêutico mais caro, o valor do frasco multidose de $350 \mathrm{ml}$ era de $R \$ 172,45$, revelando um potencial encarecimento ao custo final dos curativos.

O manejo através das coberturas especiais foi mediado através da disponibilização de oito insumos, com variados graus de custo-benefício e de densidade tecnológica. Em $20 \%$ das lesões eram utilizadas combinações de mais de uma cobertura especial, considerando que no leito de uma lesão podem coexistir características e tecidos diferentes. De modo igual, existiram lesões que, devido a sua extensão, eram empregadas mais de uma unidade de uma mesma cobertura especial, mas que os registros não permitiram calcular o percentual em que isso ocorria.

Houve predomínio do uso de três coberturas: hidrogel com alginato de cálcio e sódio, gaze rayon embebida com óleos e ácidos graxos essenciais (AGE) e alginato de cálcio em placa, utilizadas, respectivamente em $33,3 \%, 25 \%$ e em $16,7 \%$ das lesões. Em seguida encontraram-se a malha não aderente com petrolatum ( $8,3 \%$ ), a hidrofibra de carboximetilcelulose sódica e prata $(5,6 \%)$, o carvão ativado com prata em placa $(5,5 \%)$, a espuma de poliuretano impregnado com prata $(2,8 \%)$ e a bota de Unna (2,8\%). Havendo, portanto, coerência com os tipos de tecidos e demais características frequentes nos leitos das lesões já expostos na tabela 2 , tendo em vista a indicação recomendada na literatura ${ }^{(6,18,20)}$ para tais coberturas.

$\mathrm{Na}$ literatura ${ }^{(6,10,15)}$, a substância mais utilizada para $\circ$ tratamento das lesões crônicas na $A B$ foram os ácidos graxos essenciais (AGE) que estão presentes aqui no curativo Gaze de Rayon embebida com óleos, a segunda cobertura mais utilizada. Boa parte das pesquisas menciona a enorme dificuldade que se tem em se levantar o tipo de cobertura ou substância utilizada nas lesões crônicas devido a baixa qualidade e ausência de registros.

Outro ponto conveniente de ser abordado é que a existência destes insumos na UBS estudada não representa o cenário vivido em outras UBS do estado do Rio de Janeiro e tampouco da realidade brasileira. Apesar de serem imprescindíveis, a realidade evidenciada nos trabalhos da temática ${ }^{(7,13,15,18)}$ é de escassez de recursos e, quando estes estão disponíveis, se limitam a substâncias básicas, tais como os AGE, que embora tenham largo espectro de aplicação(10), baixo custo e comprovada efetividade ${ }^{(6,18,20)}$, não propiciam resultados com tanta celeridade, sobretudo em condições de 
comorbidades, tais como as presentes na população estudada.

Considerando que o cuidado das lesões é uma atribuição da atenção básica ${ }^{(12)}$, a ausência destes e de outros insumos na realidade da maioria das UBS brasileiras limita o potencial da resolutividade das unidades e dificulta a integralidade do

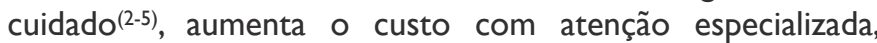
sobretudo hospitalar, e acresce o risco de incapacitações temporárias e permanentes aos sujeitos ${ }^{(16)}$.

No cenário estudado, $62,5 \%$ das coberturas especiais estiveram ausentes em algum intervalo de tempo, revelando sub oferta/desabastecimento e indisponibilidade da maioria dos insumos de cuidado às lesões crônicas. A figura 2, abaixo, descreve a relação entre demanda e oferta médias por recursos aos usuários com feridas crônicas. Ao se analisar as cinco coberturas em que a oferta foi sempre inferior a demanda, a diferença média $(\neq)$ foi de $36,1 \%$ entre demanda e oferta. Havendo, então, um distanciamento entre os insumos que são necessários e os que são disponibilizados.

A figura 2, abaixo, aponta, que houve correlação entre demanda e oferta média mensal para o hidrogel com alginato de cálcio e sódio, que também foi a substância de maior uso no serviço. Isto levantou o questionamento sobre a possibilidade do hidrogel ser a substância mais usada por ser a mais disponível, sem interrupções em seu fornecimento. Sua apresentação era em frasco multidose que após aberto e reservado em ambiente e temperatura adequados, mantinha validade de até 28 dias e era utilizado por quantidade superior a quatro usuários, a depender da extensão da ferida.

O mesmo não se repetiu com o uso da bota de Unna, que embora não tenha sofrido interrupções em seu fornecimento, seu uso seguiu específico e restrito a determinados tipos de lesões e sob prescrição médica ${ }^{(18,20)}$. Isto decorre pela linha tênue entre o potencial terapêutico e o potencial iatrogênico.
Somente uma cobertura especial - espuma de poliuretano com prata - teve oferta maior do que a demanda ( $50 \%$ além do necessário), que se repetiu ao longo dos 12 meses analisados.

Verifica-se, então, a existência de problemas com o abastecimento dos insumos, que pode estar associado a solicitação inadequada de materiais, bem como o repasse inadequado de materiais pela central de abastecimento. É interessante ressaltar que a $A B$ no município do Rio de Janeiro vem sofrendo cortes e contenções orçamentárias desde 2017 , e, portanto, acredita-se que o desabastecimento de insumos tenha relação direta com a "crise" de financiamento que os serviços de cuidados primários estão enfrentando ${ }^{(2,3)}$.

O custo médio mensal por usuário foi de $\mathrm{R} \$$ 161,93, contudo, a análise desagregada dos dados revelou a existência de opostos contrastantes, com usuários em que eram investidos $R \$ 42,40$ /mês e aqueles em que eram investidos $R \$$ I.204,64/mês, tendo em vista que o custo é variável de acordo com a cobertura utilizada, extensão da lesão e frequência de troca. No trabalho realizado por Brito e colaboradores ${ }^{(13)}$, a maioria dos usuários gerava um custo médio até $100 /$ mês, o que é um valor relativamente menor do que o encontrado no presente estudo.

Apesar das lesões crônicas serem um problema de saúde pública, conforme já abordado, poucas investigações se debruçam sobre o impacto econômico destas e seus custos ao usuário e ao sistema de saúde, especialmente nos serviços primários. Como foi visualizado, existem usuários em que os serviços têm de investir $\mathrm{R} \$ \mathrm{I} .204$ ao mês em seus tratamentos, isto considerando apenas as coberturas especiais, existindo ainda os custos que o usuário e os serviços detém com os curativos secundários, mão de obra, tratamentos medicamentosos e as consultas com especialistas - quando necessárias -.

Figura 2 - Relação entre a oferta e a demanda média mensal das coberturas especiais, ordenada pela oferta, em uma UBS do município do Rio de Janeiro/RJ, entre agosto de 2017 e julho de 2018.

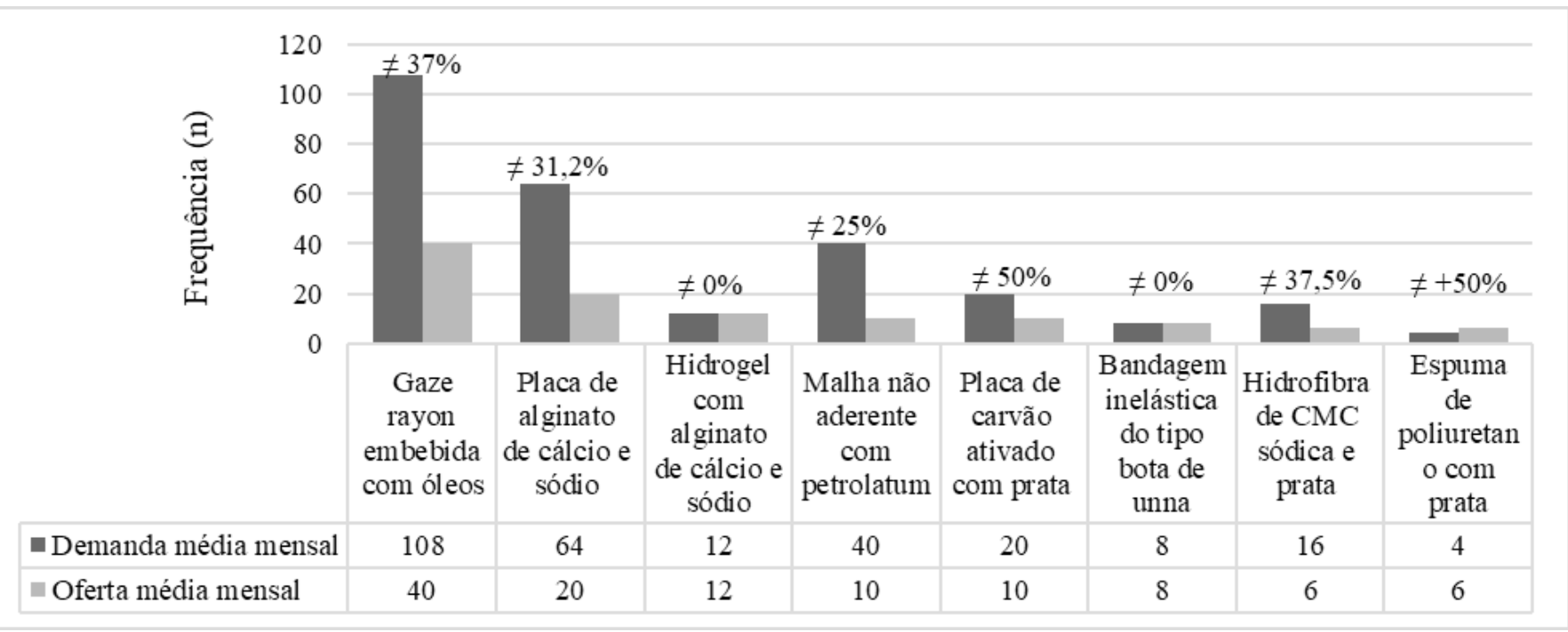




\section{CONSIDERAÇÕES FINAIS}

O presente estudo revelou tendência à ocorrência de feridas crônicas idosos, em que a idade média foi de 65,3 anos. Em $83 \%$ de todos os portadores de feridas crônicas havia o diagnóstico de alguma DCNT, sendo que $63,3 \%$ da população manifestava três ou mais diagnósticos. $\mathrm{O}$ risco social ocorria em $23,3 \%$ desta população, indicando que a análise da determinação social do processo saúde-doença, também é relevante para se debruçar sobre o fenômeno das feridas crônicas.

A análise sobre $\circ$ perfil das lesões indicou que elas ocorreram majoritariamente em membros inferiores e as alterações vasculares foram a etiologia predominante. Embora $60 \%$ das lesões apresentassem tecidos propícios à cicatrização em leito, havia evidência de estagnação cicatricial, tendo em vista que em $63,3 \%$ do total haviam indícios de colonização e ou infecção. $O$ tempo de duração foi superior a dois anos em aproximadamente $50 \%$ dos casos, podendo ser classificado como prolongado quando se observa o potencial danoso para a vida dos sujeitos, ao sistema de saúde e à sociedade.

Já o perfil de cuidados ofertados no serviço se mostrou inadequado ao acontecer preponderantemente na sala de curativos, por deter o domínio de uma única profissão, por ser centrado em tecnologias densas - coberturas especiais - e pela oferta de materiais ser continuamente interrompida. Entendese que as feridas crônicas, como fenômeno multidimensional e complexo, carecem de cuidados realizados para além de espaços com propósitos curativos, tais como consultórios e salas de curativos, merecendo cuidados multiprofissionais e interdisciplinares integrais e resolutivos. A continuidade do cuidado através do fornecimento de insumos deve ser uma responsabilidade de todos os profissionais da assistência, mas é, igualmente, um compromisso dos gestores dos serviços e dos sistemas de saúde. Estes devem ser convocados como parte crucial do processo de cuidado.

Por fim, ainda que este estudo apresente limitações amostrais e como viés a perspectiva da gestão (tendo em vista que os relatórios de gestão foram o principal material aqui analisado), fica reforçada a necessidade de fortalecimento do papel da $A B$ no manejo das $D C N T$, tendo em vista a relação destas com a prevalência de feridas crônicas. Tendo em vista que os serviços de $A B$ emergem como responsáveis pelos cuidados às feridas, especialmente as crônicas, recomenda-se a realização de outras pesquisas que explorem os modelos de atenção e o processo de trabalho direcionado aos portadores de feridas nestes serviços.

\section{REFERÊNCIAS}

I. Ministério da Saúde (Brasil). Portaria $n^{\circ} 2.436$, de 21 de setembro de 2017. Aprova a Política Nacional de Atenção Básica, estabelecendo a revisão de diretrizes para a organização da Atenção Básica, no âmbito do Sistema Único de Saúde. Diário Oficial da União 22 set 2017; Seção I.

2. Giovanella L. Atenção básica ou atenção primária à saúde?. Cad Saúde Pública [Internet]. 2018 [citado em II ago. 2019]; 34(8):e000298 |8. Disponível em: http:// dx.doi.org//0.1590/0102-3 I Ix000298/8

3. Pinto LF, Giovanella L. Do Programa à Estratégia Saúde da Família: expansão do acesso e redução das internações por condições sensíveis à atenção básica (ICSAB). Ciênc saúde coletiva [Internet]. 2018 [citado em I2 ago. 2019]; 23(6): 1903- I 4. Disponível em: http:// dx.doi.org/|0.1590/14|3-8|232018236.05592018

4. Morosini MVGC. Modelos de atenção e a saúde da família. Ia.ed. Rio de Janeiro: EPSJV/Fiocruz; 2007.

5. Mendes EV. O cuidado das condições crônicas na atenção primária à saúde: o imperativo da consolidação da estratégia da saúde da família. Brasília: Organização Pan-Americana da Saúde; 2012.

6. Coimbra FR. O tratamento de úlceras venosas na atenção primária: a utilização de tecnologias para - cuidado de enfermagem. [Monografia]. Corinto: Universidade Federal de Minas Gerais; 2012.

7. Azevedo IC, Costa RKS, Holanda CSM, Salvetti MG, Torres GV. Conhecimento de enfermeiros da estratégia saúde da família sobre avaliação e tratamento de feridas oncológicas. Rev bras cancerol. [Internet]. 2014 [citado em 12 ago. 2019]; 60(2):1 19-27. Disponível em: https://rbc.inca.gov.br/revista/index.php/revista/article/ view/476

8. Vieira CPB, Furtado AS, Almeida PCD, Luz MHBA, Pereira AFM. Prevalência e caracterização de feridas crônicas em idosos assistidos na atenção básica. Rev baiana enferm. [Internet]. 2017 [citado em 12 ago. 2019]; 3l(3):el7397. Disponível em: http://dx.doi. org/ I0.1847//rbe.v3li3.17397

9. Barbosa JTC. $\bigcirc$ gerenciamento em enfermagem na sala de curativo de uma unidade básica de saúde. [Monografia]. Niterói: Escola de Enfermagem Aurora de Afonso Costa da Universidade Federal Fluminense; 2014.

10. Evangelista DG, Magalhães ERM, Moretão DIC, Stival MM, Lima LR. Impacto das feridas crônicas na qualidade de vida de usuários da estratégia de saúde da família. Rev Enferm Cent O Min. [Internet]. 2012 [citado em 12 ago. 2019];2(2):254-63. Disponível em: http://dx.doi. org/ $10.19175 /$ recom.v0i0.15

II. Passadouro R, Sousa A, Santos C, Costa H, Craveiro I. Características e Prevalência em Cuidados de Saúde Primários das Feridas Crônicas. Rev SPDV. [Internet]. 2016 [citado em 12 ago. 2019]; 74(I):45-5I. Disponível em: https://doi.org/I0.2902I/spdv.74.I.5 I4

12. Resende NM, Nascimento T, Lopes F, Júnior A, Souza N. Cuidado de pessoas com feridas crônicas na Atenção Primária à Saúde. JMPHC [Internet]. 2017 [acesso em 12 ago. 2019]; 8(I):99-108. Disponivel em: http://www. jmphc.com.br/jmphc/article/view/27।

13. Brito CKD, Nottingham IC, Victor JF, Feitoza SMS, Silva MG,Amaral HEG. Úlcera venosa: avaliação clínica, orientações e cuidados com o curativo. Rev Rene. [Internet]. 2013 [citado em 12 ago. 2019]; 14(3):47080. Disponível em: http://www.periodicos.ufc.br/rene/ article/view/3415

14. Schleicher AT, Souza EF, Guimarães FR, Bolpato MB, Souza PR. Perfil dos pacientes portadores de feridas crônicas e avaliação do conhecimento sobre a terapêutica tópica utilizada. Sci Elec Arch. [Internet]. 2017 [citado em 12 ago. 2019]; 10(3):67-75. Disponível em: http://www.seasinop.com.br/revista/index.php?journal $=S E A \& p a g e=$ article\&op $=$ view $\&$ path\%5B\%5D $=328$

15. Barros MPL, Ferreira PJO, Maniva SJCF, Holanda R. Caracterização de feridas crônicas de um grupo de pacientes acompanhados no domicílio. Rev Interd. 
[Internet]. 2016 [citado em 12 ago. 2019]; 9(3):III. Disponível em: https://revistainterdisciplinar. uninovafapi.edu.br/index.php/revinter/article/view/926

16. Leal TS, Oliveira BG, Bomfim ES, Figueredo NL, Souza AS, Santos ISC. Percepção de pessoas com a ferida crônica. Rev enferm UFPE [Internet]. 2017 [citado em I 2 ago. 2019]; I I(3): I I56-I I62. Disponível em: DOI: I0.5205/reuol.I0544-93905-I-RV.I I 0320 I 705

17. Vieira CPB, Araújo TME. Prevalência e fatores associados a feridas crônicas em idosos na atenção básica. Rev Esc Enferm USP. [Internet]. 2018 [citado em I2 ago. 2019]; 52(I):e034I5. Disponível em: http:// dx.doi.org/I0.1590/s 1980-220x201705/3034I5

18. Stambassi G. Processo de trabalho em enfermagem: cuidado a pessoas com feridas crônicas na atenção primária à saúde. [Dissertação] Juiz de Fora: Faculdade de Enfermagem da Universidade Federal de Juiz de Fora; 2015.
19. Ministério da Saúde (Brasil). Parecer TécnicoCientífico: Avaliação de Múltiplas Tecnologias em Feridas Crônicas e Queimaduras. Brasília: Ministério da Saúde; 201 I.

20. Campos MGCA, De Souza ATO, Vasconcelos JMB, De Lucena SAP, Gomes SKA. Feridas complexas e estomias: aspectos preventivos e manejo clínico. João Pessoa: Ideia; 2016.
Recebido: 2019-08-30

Aceito: $2019-11-23$ 